\title{
BMJ Open Insurance status and 1-year outcomes of stroke and transient ischaemic attack: a registry-based cohort study in China
}

\author{
Hong-Qiu Gu, ${ }^{1,2}$ Zi-Xiao Li, ${ }^{1,3}$ Xing-Quan Zhao, ${ }^{1,3}$ Li-Ping Liu, ${ }^{4,5}$ Hao Li, ${ }^{1,2}$ \\ Chun-Juan Wang, ${ }^{2,6}$ Xin Yang, ${ }^{1,2}$ Zhen-Zhen Rao, ${ }^{7}$ Chun-Xue Wang, ${ }^{3,8}$ \\ Yue-Song Pan, ${ }^{1,2}$ Yi-Long Wang, ${ }^{1,2}$ Yong-Jun Wang, ${ }^{1,2,3}$ on behalf of China National \\ Stroke Registries
}

To cite: Gu H-Q, Li Z-X, Zhao $X-Q$, et al. Insurance status and 1-year outcomes of stroke and transient ischaemic attack: a registry-based cohort study in China. BMJ Open 2018;8:e021334. doi:10.1136/ bmjopen-2017-021334

- Prepublication history and additional material for this paper are available online. To view these files, please visit the journal online (http://dx.doi. org/10.1136/bmjopen-2017021334).

$\mathrm{H}-\mathrm{QG}$ and $\mathrm{Z}-\mathrm{XL}$ contributed equally.

Received 27 December 2017 Revised 21 April 2018 Accepted 1 June 2018

Check for updates

For numbered affiliations see end of article.

Correspondence to

Dr Yong-Jun Wang;

yongjunwang1962@gmail.com

\section{ABSTRACT}

Objective Although more than $95 \%$ of the population is insured by urban or rural insurance programmes in China, little research has been done on insurance-related outcome disparities for patients with acute stroke and transient ischaemic attack (TIA). This study aimed to examine the relationship between insurance status and 1-year outcomes for patients with stroke and TIA. Methods We abstracted 24941 patients with acute stroke and TIA from the China National Stroke Registry II. Insurance status was categorised as Urban Basic Medical Insurance Scheme (UBMIS), New Rural Cooperative Medical Scheme (NRCMS) and self-payment. The relationship between insurance status and 1-year outcomes, including all-cause death, stroke recurrence and disability, was analysed using the shared frailty model in the Cox model or generalised estimating equation with consideration of the hospital's cluster effect.

Results About $50 \%$ of patients were covered by UBMIS, $41.2 \%$ by NRCMS and $8.9 \%$ by self-payment. Compared with patients covered by UBMIS, patients covered by NRCMS had a significantly higher risk of all-cause death (9.7\% vs $8.6 \%$, adjusted HR: 1.32 (95\% $\mathrm{Cl} 1.17$ to 1.48$)$, $\mathrm{p}<0.001)$, stroke recurrence $(7.2 \%$ vs $6.5 \%$, adjusted HR: $1.12(95 \% \mathrm{Cl} 1.11$ to 1.37$), \mathrm{p}<0.001)$ and disability (32.0\% vs $26.3 \%$, adjusted OR: 1.29 (95\% Cl 1.21 to 1.39), $\mathrm{p}<0.001)$. Compared with patients covered by UBMIS, selfpayment patients had a similar risk of death and stroke recurrence but a higher risk of disability.

Conclusions Patients with stroke and TIA demonstrated differences in 1-year mortality, stroke recurrence and disability between urban and rural insurance groups in China.

\section{INTRODUCTION}

Studies in the USA and Canada have suggested that insurance is an important independent indicator of health outcomes. ${ }^{1-3}$ Since 1994, China has undertaken a series of healthcare insurance initiatives. ${ }^{45}$ By 2011, 95\% of the population was insured by the New Rural Cooperative Medical Scheme (NRCMS), Urban Resident Basic Medical Insurance (URBMI) or Urban Employee Basic Medical Insurance (UEBMI). ${ }^{4}$ The target populations

\section{Strengths and limitations of this study}

We provide a large-scale, nationwide, prospective registry project-based study to explore the association between insurance status and 1-year outcome for patients with stroke and transient ischaemic attack.

- High-quality data obtained through systematic and standardised approach and statistical methods considering the cluster effect of the hospital and competing risk of stroke were used to draw the conclusions.

- The sampling method we used did not strictly represent nationwide hospitals.

- One-year outcomes obtained by telephone follow-up were not an accurate measurement.

- Unmeasured factors including lifestyle issues or even ethnic/genetic variations may affect the results.

of NRCMS, URBMI and UEBMI are rural residents, urban residents and urban employees, respectively. Both inpatients and outpatients are covered by each insurance programme. Compared with NRCMS, the insurance premium and reimbursement rate are much higher for URBMI and UEBMI. ${ }^{4}$ Although health insurance coverage can reduce the financial burden and improve health service utilisation, ${ }^{6}$ different insurance programmes may have different effects on health outcomes due to different restrictions on access to healthcare. Some studies have suggested that better health insurance predicts more favourable stroke outcomes. ${ }^{7-9}$ In China, some studies also suggested that urban health insurances are related to better hypertension management and less major adverse cardiovascular events. However, these studies were focused on cardiovascular diseases, ${ }^{10-12}$ or limited only to prevalence or mortality of stroke,${ }^{1314}$ or too early from the accomplishment of the series of healthcare insurance initiatives. $^{14}$ 
Stroke is the second leading cause of death and a major cause of long-term disability worldwide. ${ }^{15} 16$ China has experienced an increasing stroke burden over the last 30 years, especially in rural areas. ${ }^{17}$ Stroke is the leading cause of death in rural areas and the third leading cause of death in urban cities in China. ${ }^{18}$ In rural areas, the population is covered mainly by rural insurance groups. However, it is unclear whether insurance-related outcome disparities exist for patients with stroke and transient ischaemic attack (TIA) in China. Therefore, we examined the relationship between insurance status and 1-year outcomes for patients with stroke and TIA. Ischaemic and haemorrhagic types of stroke and TIA were also analysed to provide a comprehensive picture of cerebrovascular disease outcomes across different insurance groups.

\section{METHODS}

\section{Study design of the China National Stroke Registry II}

The design of the China National Stroke Registry II (CNSR II) study has been described previously. ${ }^{1920}$ Briefly, CNSR II is a nationwide, hospital-based, prospective cohort study initiated in 2012 by the Ministry of Health to assess stroke care delivery and 1-year outcomes to identify fields that need further improvement when compared with CNSR I in 2007. A total of 219 secondary or tertiary public hospitals were selected by the steering committee using the same criteria applied in CNSR I. ${ }^{21}$ Each participant signed an informed consent before his/her participation in the study.

\section{Participant enrolment and study population}

Patients were recruited consecutively from all 219 hospitals from June 2012 to January 2013. Inclusion criteria included (1) $\geq 18$ years of age; (2) diagnosis within 7 days of the index event of acute ischaemic stroke, TIA, spontaneous intracerebral haemorrhage or subarachnoid haemorrhage confirmed by brain CT or MRI; (3) direct hospital admission from a physician's clinic or emergency department; and (4) written informed consent offered by the patient or his/her legally authorised representative.

\section{Data collection and abstraction}

Trained research coordinators at each hospital checked the medical records daily to identify, obtain informed consent and recruit consecutive patients. Data on prestroke modified Rankin Scale ${ }^{22}$ (mRS, with scores ranging from 0 to 6 , with a score of 0 indicating no disability and higher scores indicating more severe disability) and National Institutes of Health Stroke Scale ${ }^{23}$ (NIHSS, with scores ranging from 0 to 42 , with higher scores indicating a more severe deficit) were obtained from interviews with patients or their legal representative. Other data extracted from the medical records included patient demographics, health insurance status, education level (classified into three levels: elementary or below ( $\leq 6$ years), middle school (7-9 years), and high school or above ( $\geq 10$ years $)$ ), household income (classified into two categories based on per capita monthly income: $\leq ¥ 1000$ and $>¥ 1000$ ), smoking, drinking, vascular risk factors, usage of medications, selected laboratory tests and discharge status. Vascular risk factors included history of stroke/TIA, hypertension, diabetes mellitus, dyslipidaemia, atrial fibrillation, coronary heart disease, myocardial infarction and peripheral vascular disease. They were defined as a medical chart-confirmed history, usage of corresponding medications before stroke onset, or a physician's diagnosis according to the findings of physical or auxiliary examinations during hospitalisation.

\section{Patient and public involvement}

Patients and the public were not directly involved in the development of, recruitment to and conduct of the study. The results of the study will be disseminated to the patients and the public through social media.

\section{Insurance status}

During the data collection stage, URBMI and UEBMI were categorised as Urban Basic Medical Insurance Scheme (UBMIS) in the case report form. Since only 77 $(0.3 \%)$ patients were insured by commercial insurance in CNSR II, we limited our study population to participants with NRCMS, UBMIS and self-payment.

\section{Clinical outcomes}

Patients were contacted by telephone at 3, 6 and 12 months after stroke onset by trained research personnel who followed standardised scripts to collect information on death, stroke recurrence and disability (defined as mRS score $>2$ ). During the follow-up periods, stroke recurrences associated with rehospitalisation were sourced to the attended hospitals to ensure reliable diagnosis. In case of a suspected recurrent cerebrovascular event without hospitalisation, judgement was made by the research coordinators together with the principal investigator.

\section{Loss to follow-up}

A patient was regarded to have been lost to follow-up if consent to participate was withdrawn or no contact could be made after three telephone calls each day (morning, afternoon and early evening) for a working week at each follow-up point. Our report was based on patients with at least one set of follow-up information obtained at 3, 6 and 12 months, or with completed data of in-hospital outcomes. We used last observation carried forward to handle missing data, which means the outcomes of patients lost to follow-up would be imputed by the latest available outcomes from in-hospital to 12-month follow-up.

\section{Statistical analysis}

For patients' baseline characteristics, continuous and categorical variables were reported as mean $\pm \mathrm{SD}$ or frequencies (percentages), respectively. For the NIHSS, we reported both median (IQR) and categorised frequencies (percentages). Comparisons of continuous variables between groups were performed by analysis of variance or 
Kruskal-Wallis test when appropriate, and of categorical variables by $\chi^{2}$ test.

For all-cause death and stroke recurrence, the Cox proportional hazard model was used to analyse the relationship between health insurance status and 1-year clinical outcomes in both unadjusted and multivariate-adjusted models. We used the shared frailty model of the Cox model in which hospitals were incorporated into the SAS PHREG procedure's random statement and regarded as independent and identically distributed random variables. ${ }^{24}$ Considering death is the competing risk of stroke recurrence, we used the competing-risks analysis of Fine and $\mathrm{Gray}^{25}$ to assess the association between insurance status and stroke recurrence. For disability, we used a generalised estimating equation model to control the cluster effect of the hospital to get a more precise estimation. ${ }^{26}$ To achieve model parsimony and stability, we first used backward selection methods with the default alpha of 0.05 to select covariates from an overall list of candidate risk factors for each outcome, and then adjusted for the selected covariates in multivariate models. The candidate risk factors affecting clinical outcomes were selected on the basis of the literature reviews, clinical plausibility and variables collected in the database. These candidate risk factors included age, education level, household income, mRS score before stroke onset, NIHSS score on admission, smoking, drinking, previous stroke or TIA, hypertension, diabetes mellitus, dyslipidaemia, atrial fibrillation, coronary heart disease, myocardial infarction, heart failure, peripheral vascular disease, hospital level, antiplatelets and anticoagulants.

To be more specific, we also assessed the association between insurance status and 1-year outcomes stratified by acute cerebrovascular disease types. We reanalysed the data on ischaemic stroke, TIA and intracerebral haemorrhage separately. We did not analyse patients with subarachnoid haemorrhage because there were only a few patients in this stratum.

All $\mathrm{p}$ values are two-sided, with $\mathrm{p}<0.05$ considered statistically significant. All statistical analyses were performed using SAS V.9.4 software.

\section{RESULTS}

The CNSR II study enrolled a total of 25108 patients with acute cerebrovascular diseases, of whom 77 patients with commercial insurance were excluded. The remaining 24941 patients were included in our data analysis.

\section{Demographical and clinical characteristics}

Among the 24941 analysed patients, 12445 (49.9\%) were covered by UBMIS, $10283(41.2 \%)$ were covered by NRCMS and 2213 (8.9\%) were self-payment. Patients covered by UBMIS were older, and had higher education, income and prevalence of vascular risk factors, such as history of stroke/TIA, heart failure or myocardial infarction, diabetes mellitus, dyslipidaemia, and atrial fibrillation, but had lower NIHSS scores compared with those covered by NRCMS. Ischaemic stroke was the primary type of cerebrovascular disease in patients in all three insurance groups. However, the proportion of TIA was higher in patients covered by UBMIS, and intracerebral haemorrhage was more prevalent in patients covered by NRCMS $(p<0.0001)$. Other details are shown in table 1.

\section{Insurance status and 1-year clinical outcomes}

During the 1-year follow-up period after stroke onset, $259(9.1 \%)$ had all-cause deaths, $1674(6.7 \%)$ had stroke recurrence and $8419(33.8 \%)$ had disability. The Kaplan-Meier curves were significantly different among the different insurance programme groups for all-cause death (figure 1; log-rank, $\mathrm{p}<0.0001$ ) but not for stroke recurrence (figure 2; Gray test, $\mathrm{p}=0.0523$ ). The number and percentage of outcomes and missing values at every period from in-hospital to 12-month visit are reported in online supplementary table 1.

In the Cox model analysis, we found that compared with inpatients covered by UBMIS, those covered by NRCMS were associated with a significantly higher risk of 1-year mortality $(9.7 \%$ vs $8.6 \%$, crude HR: $1.32,95 \%$ CI 1.18 to $1.46, \mathrm{p}<0.0001)$. After adjusting for the relevant covariates, the risk remained 32\% higher (adjusted HR: 1.32, $95 \%$ CI 1.17 to $1.48, \mathrm{p}<0.0001)$. We also found a greater higher risk of 1-year stroke recurrence in the crude analysis $(7.2 \%$ vs $6.5 \%$, crude HR: 1.11 (95\% CI 1.01 to 1.23 ), $\mathrm{p}=0.0357$ ) and in the adjusted results (adjusted HR: 1.12, 95\% CI 1.11 to $1.37, \mathrm{p}<0.0001)$. With regard to 1 -year disability, patients covered by NRCMS also showed a higher risk in the crude analysis $(32.0 \%$ vs $26.3 \%$, crude OR: 1.32 , $95 \%$ CI 1.25 to $1.41, \mathrm{p}<0.0001)$ and in the adjusted analysis (adjusted OR: 1.29 , 95\% CI 1.21 to 1.39 , p<0.0001). Self-payment insurance patients had a similar risk of all-cause mortality as patients covered by UBMIS (adjusted HR: $1.18,95 \%$ CI 0.99 to $1.42, p=0.0702$ ), and stroke recurrence (adjusted HR: $1.03,95 \%$ CI 0.86 to $1.25, p=0.7261$ ), but higher risk of disability (adjusted OR: 1.27, 95\% CI 1.14 to $1.42, \mathrm{p}<0.0001$ ) (table 2 ).

\section{Stratified analysis}

The stratified analysis in table 3 showed that 1-year outcome risks varied among different cerebrovascular disease types. Among patients with ischaemic stroke, compared with patients covered by UBMIS, patients covered by NRCMS had a $34 \%$ higher risk of all-cause death (adjusted HR: $1.34,95 \%$ CI 1.18 to $1.54, \mathrm{p}<0.0001$ ), $34 \%$ higher risk of stroke recurrence (adjusted HR: $1.34,95 \%$ CI 1.20 to $1.51, \mathrm{p}<0.0001)$ and $30 \%$ higher risk of disability (adjusted HR: $1.30,95 \%$ CI 1.21 to 1.41 , $\mathrm{p}<0.0001)$. However, we did not see patients covered by NRCMS had a higher risk of all-cause death, stroke recurrence and disability among other types of cerebrovascular diseases, except for the higher risk of disability among patients with intracerebral haemorrhage (adjusted OR: $1.27,95 \%$ CI 1.06 to $1.52, \mathrm{p}=0.0112)$. Self-payment patients had a similar risk of 1-year outcomes to patients covered by UBMIS among all types of cerebrovascular diseases. 
Table 1 Baseline characteristics by insurance status

\begin{tabular}{|c|c|c|c|c|}
\hline Variables & $\begin{array}{l}\text { UBMIS } \\
(n=12445)\end{array}$ & $\begin{array}{l}\text { NRCMS } \\
(n=10283)\end{array}$ & $\begin{array}{l}\text { Self-payment } \\
(\mathrm{n}=2213)\end{array}$ & $P$ values \\
\hline Age, mean \pm SD & $65.8 \pm 12.1$ & $63.1 \pm 11.7$ & $61.3 \pm 13.1$ & $<0.0001$ \\
\hline Male & $8320(66.9)$ & $5838(56.8)$ & $1454(65.7)$ & $<0.0001$ \\
\hline Education & & & & $<0.0001$ \\
\hline Elementary or below & $3926(31.5)$ & $6480(63.0)$ & $1040(47.0)$ & \\
\hline Middle school & $3131(25.2)$ & 2215 (21.5) & $483(21.8)$ & \\
\hline High school or above & 5388 (43.3) & $1588(15.4)$ & $690(31.2)$ & \\
\hline Family monthly income per capita & & & & $<0.0001$ \\
\hline$\leq 1000$ & $938(7.5)$ & $4088(39.8)$ & $333(15.0)$ & \\
\hline$>1000$ & $8151(65.5)$ & $3399(33.1)$ & $894(40.4)$ & \\
\hline Unknown & $3356(27.0)$ & $2796(27.2)$ & $986(44.6)$ & \\
\hline NIHSS score, median (IQR) & $3.0(1.0-6.0)$ & $4.0(2.0-8.0)$ & $4.0(2.0-7.0)$ & $<0.0001$ \\
\hline NIHSS score, categories & & & & $<0.0001$ \\
\hline $0-4$ & $6540(52.6)$ & $4683(45.5)$ & $952(43.0)$ & \\
\hline $5-14$ & $4991(40.1)$ & $4642(45.1)$ & $1088(49.2)$ & \\
\hline$\geq 15$ & $914(7.3)$ & $958(9.3)$ & $173(7.8)$ & \\
\hline Cerebrovascular disease & & & & $<0.0001$ \\
\hline Ischaemic stroke & 9939 (79.9) & 7654 (74.4) & $1748(79.0)$ & \\
\hline Transient ischaemic attack & $1241(10.0)$ & $789(7.7)$ & $125(5.6)$ & \\
\hline Intracerebral haemorrhage & 1107 (8.9) & 1639 (15.9) & $296(13.4)$ & \\
\hline Subarachnoid haemorrhage & $95(0.8)$ & 169 (1.6) & $33(1.5)$ & \\
\hline Unspecified & $63(0.5)$ & $32(0.3)$ & $11(0.5)$ & \\
\hline Smoking & $5473(44.0)$ & 4260 (41.4) & 987 (44.6) & 0.0002 \\
\hline Drinking & $3752(30.1)$ & $2858(27.8)$ & $713(32.2)$ & $<0.0001$ \\
\hline Previous stroke/TIA & $4828(38.8)$ & $3085(30.0)$ & $592(26.8)$ & $<0.0001$ \\
\hline Heart failure/MI & $603(4.8)$ & $294(2.9)$ & $75(3.4)$ & $<0.0001$ \\
\hline Hypertension & $9580(77.0)$ & $7788(75.7)$ & $1671(75.5)$ & 0.0570 \\
\hline Diabetes mellitus & 3605 (29.0) & $1877(18.3)$ & $491(22.2)$ & $<0.0001$ \\
\hline Dyslipidaemia & $4374(35.1)$ & $3213(31.2)$ & $580(26.2)$ & $<0.0001$ \\
\hline $\mathrm{AF}$ & $1092(8.8)$ & $677(6.6)$ & $185(8.4)$ & $<0.0001$ \\
\hline Peripheral artery disease & $649(5.2)$ & $379(3.7)$ & $118(5.3)$ & $<0.0001$ \\
\hline mRS score & & & & $<0.0001$ \\
\hline $0-2$ & $11117(89.3)$ & 9398 (91.4) & 1977 (89.3) & \\
\hline $3-5$ & $1328(10.7)$ & $885(8.6)$ & $236(10.7)$ & \\
\hline Antiplatelets & 9169 (73.7) & 7111 (69.2) & $1481(66.9)$ & $<0.0001$ \\
\hline Anticoagulants among AF & 107/1092 (9.8) & $80 / 677$ (11.8) & $17 / 185(9.2)$ & 0.3391 \\
\hline Tertiary hospital & 9795 (78.7) & 5655 (55.0) & 1747 (78.9) & $<0.0001$ \\
\hline
\end{tabular}

AF, atrial fibrillation; MI, myocardial infarction; mRS, modified Rankin Scale; NIHSS, National Institutes of Health Stroke Scale; NRCMS, New Rural Cooperative Medical Scheme; TIA, transient ischaemic attack; UBMIS, Urban Basic Medical Insurance Scheme.

\section{DISCUSSION}

This national registry study demonstrated that health insurance status is significantly associated with 1-year outcomes for patients with stroke and TIA in China. Compared with patients covered by UBMIS, patients covered by NRMCS have 32\% higher risk of all-cause death, $12 \%$ higher risk of stroke recurrence and more than $29 \%$ greater risk of disability. In addition, we found that the risk for unfavourable outcomes was mainly among patients with ischaemic stroke.

China has made an impressive achievement in both the scale of coverage and the speed of expansion of its health insurance system. Among the above-mentioned insurance programmes in China, the UEBMI covers more than $95 \%$ 


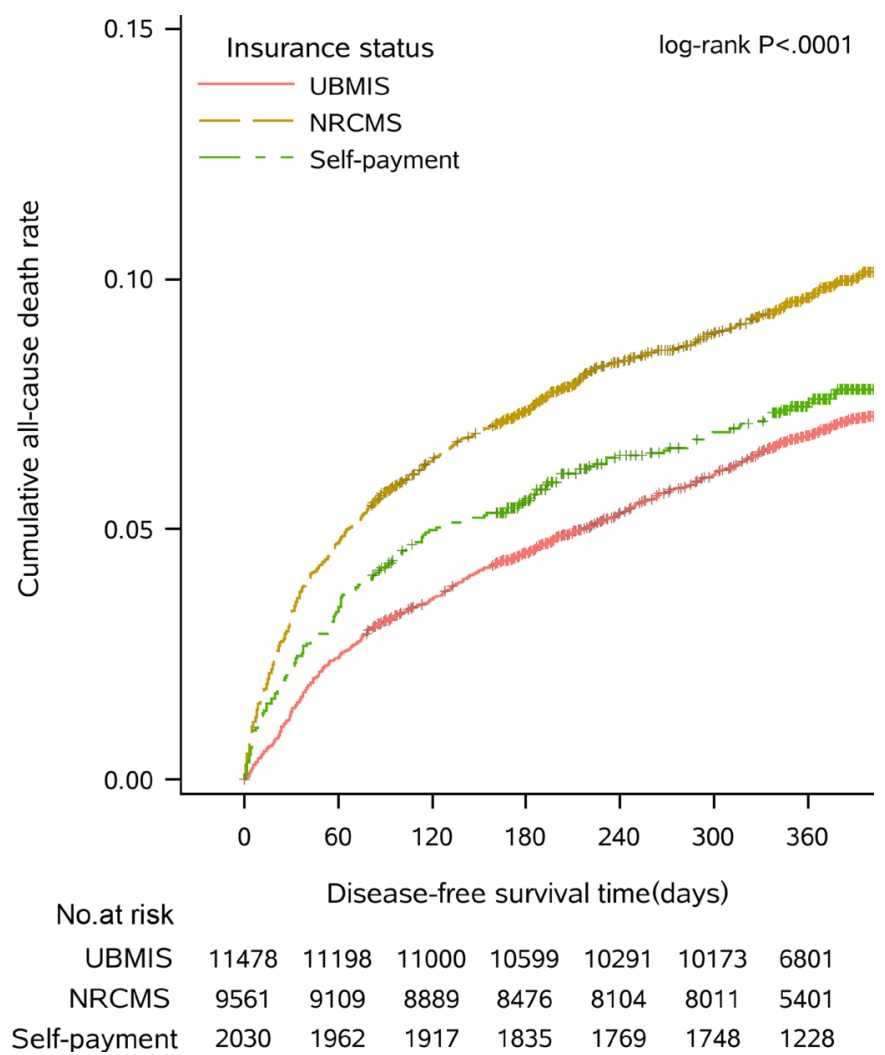

Figure 1 Cumulative Kaplan-Meier estimates of the time to all-cause death. NRCMS, New Rural Cooperative Medical Scheme; UBMIS, Urban Basic Medical Insurance Scheme.

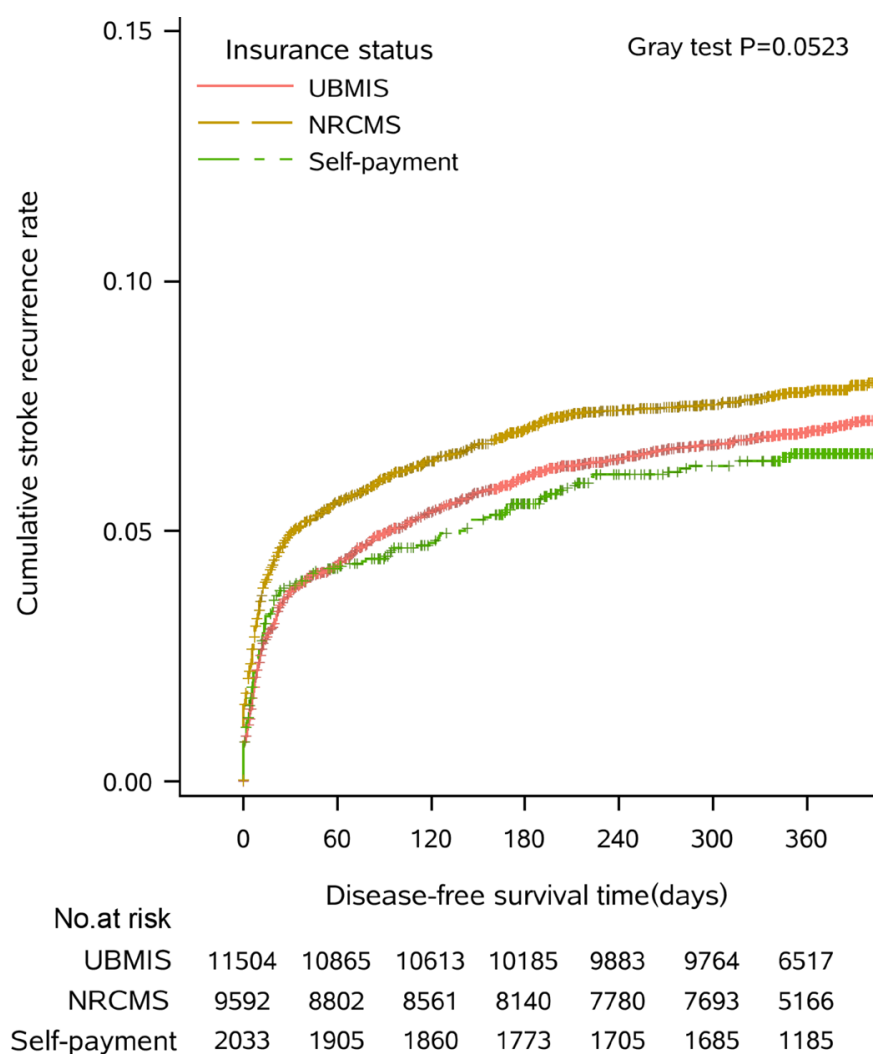

Figure 2 Cumulative Kaplan-Meier estimates of the time to stroke recurrence. NRCMS, New Rural Cooperative Medical Scheme; UBMIS, Urban Basic Medical Insurance Scheme. of urban employees and retired employees, the URBMI also covers more than $95 \%$ of urban residents, and the NRCMS covers almost $99 \%$ of rural residents. UEBMI and URBMI compose the UBMIS. NRCMS and UBMIS have covered almost the entire Chinese population, leaving only a small percentage of self-payment and commercial insurance. ${ }^{27}$ Therefore, we abstracted patients with NRCMS, UBMIS and self-payment. However, due to the differences in insurance premiums and reimbursement rates, differences in health service utilisation and disease outcomes exist between NRCMS and UBMIS. So we emphasised comparisons between NRCMS and UBMIS. In addition to the disparities between NRCMS and UBMIS, we found that the self-payment group was not significantly associated with poor outcomes compared with the UBMIS group. One possible reason is that some of the self-payment patients with NRCMS or UBMIS transferred to other provincial hospitals, who could report self-payment insurance status. However, we do not have enough data to evaluate this in detail.

Most of the previous studies ${ }^{72^{28-31}}$ have found that insurance status is an independent indicator of stroke outcome. Some previous studies demonstrated that patients with stroke with health insurance were less likely to experience unfavourable outcomes than those without health insurance, ${ }^{7928}$ and some other studies have shown that patients with private health insurance had a lower risk for unfavourable outcomes than those with other health insurance. ${ }^{30} 31$ Currently, most private health insurance programmes provide supplemental insurance products to complement public health insurance programmes. The higher rate and broader range of reimbursements may lead to greater utilisation of health service and better health outcomes. ${ }^{21}$ Our data showed that patients covered by NRCMS had less use of antiplatelets and are less hospitalised to tertiary hospitals, and these may be largely due to the lower reimbursement of NRCMS. In addition, patients covered by NRCMS had generally disadvantaged social economic status (eg, less educated, lower income) and more severe stroke disease (higher NIHSS score, more intracerebral haemorrhage). Studies have reported that lower social economic status and higher NIHSS scores have more severe deficits and higher mortality risk. ${ }^{32}{ }^{33}$ This may also partly explain the outcome difference between UBMIS and NRCMS.

Numerous studies have indicated that health insurance can reduce disparities in access to care and health outcomes. ${ }^{163435}$ Researchers believe that fragmentation in social health insurance schemes is a major factor for inequitable access to healthcare and financial protection. ${ }^{27} 3136$ Many existing studies have demonstrated the association between health insurance and patients' outcomes. ${ }^{1}$ One reason is that the fragmentation of health insurance means poor health insurance funding pools, which leads to differential benefit packages, and unequal access to needed care and financial protection. ${ }^{3738}$ Rural populations have more restricted access to healthcare and also have a higher economic burden than 
Table 2 Association between risk factors and 1-year outcomes

\begin{tabular}{|c|c|c|c|c|c|c|}
\hline \multirow[b]{2}{*}{ Variables } & \multirow[b]{2}{*}{ Patients (n) } & \multirow{2}{*}{$\begin{array}{l}\text { 1-year outcomes, } \\
\mathrm{n}(\%)\end{array}$} & \multicolumn{2}{|l|}{ Crude results } & \multicolumn{2}{|l|}{ Adjusted results } \\
\hline & & & HR/OR (95\% Cl) & $P$ values & HR/OR (95\% Cl) & $P$ values \\
\hline \multicolumn{7}{|l|}{ All-cause death* } \\
\hline \multicolumn{7}{|l|}{ Insurance status } \\
\hline NRCMS & 10283 & $994(9.7)$ & 1.32 (1.18 to 1.46$)$ & $<0.0001$ & 1.32 (1.17 to 1.48$)$ & $<0.0001$ \\
\hline Self-payment & 2213 & $191(8.6)$ & 1.07 (0.90 to 1.28$)$ & 0.4349 & 1.18 (0.99 to 1.42$)$ & 0.0702 \\
\hline UBMIS & 12445 & $1074(8.6)$ & Ref & & Ref & \\
\hline \multicolumn{7}{|l|}{ Stroke recurrence $†$} \\
\hline \multicolumn{7}{|l|}{ Insurance status } \\
\hline NRCMS & 10283 & 739 (7.2) & 1.11 (1.01 to 1.23$)$ & 0.0357 & 1.12 (1.11 to 1.37 ) & $<0.0001$ \\
\hline Self-payment & 2213 & $129(5.8)$ & 0.91 (0.75 to 1.09 ) & 0.3003 & 1.03 (0.86 to 1.25$)$ & 0.7261 \\
\hline UBMIS & 12445 & $806(6.5)$ & Ref & & Ref & \\
\hline \multicolumn{7}{|l|}{ Disability } \\
\hline \multicolumn{7}{|l|}{ Insurance status } \\
\hline NRCMS & 9398 & 3011 (32.0) & $1.32(1.25$ to 1.41$)$ & $<0.0001$ & 1.29 (1.21 to 1.39$)$ & $<0.0001$ \\
\hline Self-payment & 1977 & 619 (31.3) & 1.28 (1.15 to 1.42$)$ & $<0.0001$ & 1.27 (1.14 to 1.42$)$ & $<0.0001$ \\
\hline UBMIS & 11117 & 2919 (26.3) & Ref & & Ref & \\
\hline
\end{tabular}

*Adjusted for age, mRS score, family monthly income per capita, smoking, drinking, NIHSS score on administration, cerebrovascular disease type, previous stroke or TIA, heart failure/myocardial infarction, dyslipidaemia, hospital level, antiplatelets and anticoagulants in the Cox model with consideration of the hospital's cluster effect.

†Adjusted for age, mRS score, family monthly income per capita, drinking, NIHSS score on administration, previous stroke or TIA, heart failure/myocardial infarction, hypertension, diabetes mellitus, hospital level, antiplatelets and anticoagulants in the Cox model with consideration of the hospital's cluster effect.

$\ddagger$ Adjusted for age, sex, education, mRS score, cerebrovascular diseases type, smoking, drinking, heart failure or myocardial infarction, diabetes mellitus, hospital level, antiplatelets and anticoagulants in the generalised equation estimation model.

mRS, modified Rankin Scale; NIHSS, National Institutes of Health Stroke Scale; NRCMS, New Rural Cooperative Medical Scheme; Ref, reference; TIA, transient ischaemic attack; UBMIS, Urban Basic Medical Insurance Scheme.

urban residents in China. ${ }^{27}$ Our results were consistent with evidence from a cardiovascular disease research in China. ${ }^{11}$

Our findings will provide convictive evidence and valuable insight into the promotion of the universal medical insurance. After the release of 'Basic medical insurance for urban and rural residents in Beijing' in December 2017, Beijing has merged UBMIS and NRCMS, and the universal medical insurance has been available for all urban and rural residents in Beijing since 1 January 2018. The reimbursement rate for inpatients is as high as $80 \%$, and the financial assistance increased by $40 \% \cdot{ }^{39}$ All these efforts will pave the way for equal access to healthcare, especially for rural residents.

The strength of this study is that it is a large-scale, nationwide, prospective, registry project-based study that explores the association between insurance status and 1-year outcome for patients with stroke and TIA. Using high-quality data obtained through systematic and standardised approach and statistical methods considering the cluster effect of the hospital and competing risk of stroke, we got a more precise estimation. To test the targeted association of different cerebrovascular disease types, we also used stratified analysis, and the association mainly remained in ischaemic stroke.
This study had some limitations. First, the sampling method we used did not strictly represent nationwide hospitals. Therefore, caution is needed in interpreting the data. Given the vast number of hospitals in China, it would be practically impossible to conduct a genuinely representative study. Being cognizant of this limitation, the steering committee selected hospitals with the consideration of both representations of hospitals and feasibility of the study in the design phase. In the analysis phase, we considered a hospital as a random cluster factor and we adjusted hospital level in multiregression models. These measures helped improve the robustness of the study. Second, 1-year outcomes obtained by telephone follow-up were not an accurate measurement. However, centralised follow-up conducted by trained research personnel with standardised scripts would minimise the inter-rater disagreement, and this is also a practical approach to conduct such a large-scale study. Third, patients lost to follow-up may substantially bias the results if the characteristics are different between missing and non-missing outcomes groups. However, the supplemental analysis showed that there was no significant difference between these two groups of population except for education (online supplementary table 2). Last, insurance is nearly completely depending on the sociodemographic feature 
Table 3 Association between risk factors and 1-year outcomes stratified by cerebrovascular disease type

\begin{tabular}{|c|c|c|c|c|c|c|}
\hline \multirow[b]{2}{*}{ Variables } & \multirow[b]{2}{*}{ Patients (n) } & \multirow{2}{*}{$\begin{array}{l}\text { 1-year outcomes, } n \\
(\%)\end{array}$} & \multicolumn{2}{|l|}{ Crude results } & \multicolumn{2}{|l|}{ Adjusted results } \\
\hline & & & HR/OR (95\% Cl) & $P$ values & HR/OR (95\% Cl) & $P$ values \\
\hline \multicolumn{7}{|l|}{ All-cause death ${ }^{*}$} \\
\hline NRCMS & 7654 & $669(8.7)$ & 1.26 (1.11 to 1.42$)$ & 0.0002 & 1.34 (1.18 to 1.54$)$ & $<0.0001$ \\
\hline Self-payment & 1748 & $146(8.4)$ & 1.06 (0.87 to 1.30$)$ & 0.5407 & $1.20(0.98$ to 1.47$)$ & 0.0703 \\
\hline NRCMS & 789 & $18(2.3)$ & 1.10 (0.61 to 2.01$)$ & 0.7487 & 1.17 (0.59 to 2.30$)$ & 0.6588 \\
\hline Self-payment & 125 & $3(2.4)$ & $1.13(0.34$ to 3.75$)$ & 0.8358 & 1.23 (0.36 to 4.22$)$ & 0.7381 \\
\hline UBMIS & 1241 & $28(2.3)$ & Ref & & Ref & \\
\hline \multicolumn{7}{|c|}{ Intracerebral haemorrhage } \\
\hline \multicolumn{7}{|l|}{ Stroke recurrence $†$} \\
\hline \multicolumn{7}{|l|}{ Ischaemic stroke } \\
\hline NRCMS & 7654 & $557(7.3)$ & 1.16 (1.03 to 1.30$)$ & 0.0115 & 1.34 (1.20 to 1.51$)$ & $<0.0001$ \\
\hline Self-payment & 1748 & $103(5.9)$ & 0.93 (0.76 to 1.15$)$ & 0.4935 & $1.06(0.86$ to 1.31$)$ & 0.5795 \\
\hline UBMIS & 9939 & 629 (6.3) & Ref & & Ref & \\
\hline \multicolumn{7}{|c|}{ Transient ischaemic attack } \\
\hline NRCMS & 789 & $46(5.8)$ & 0.88 (0.62 to 1.27$)$ & 0.5000 & 0.94 (0.65 to 1.37$)$ & 0.7558 \\
\hline Self-payment & 125 & $9(7.2)$ & 1.14 (0.57 to 2.26$)$ & 0.7127 & 1.05 (0.54 to 2.02$)$ & 0.8931 \\
\hline UBMIS & 1241 & $82(6.6)$ & Ref & & Ref & \\
\hline NRCMS & 6968 & $2185(31.4)$ & 1.24 (1.16 to 1.33$)$ & $<0.0001$ & 1.30 (1.21 to 1.41$)$ & $<0.0001$ \\
\hline Self-payment & 1552 & $488(31.4)$ & 1.25 (1.11 to 1.40$)$ & 0.0002 & $1.33(1.18$ to 1.51$)$ & $<0.0001$ \\
\hline UBMIS & 8820 & $2373(26.9)$ & Ref & & Ref & \\
\hline \multicolumn{7}{|c|}{ Transient ischaemic attack } \\
\hline NRCMS & 775 & $59(7.6)$ & $1.08(0.77$ to 1.53$)$ & 0.6437 & $1.31(0.90$ to 1.91$)$ & 0.1649 \\
\hline Self-payment & 120 & $12(10.0)$ & 1.46 (0.77 to 2.76$)$ & 0.2409 & 1.74 (0.90 to 3.36$)$ & 0.0992 \\
\hline UBMIS & 1218 & $86(7.1)$ & Ref & & Ref & \\
\hline \multicolumn{7}{|c|}{ Intracerebral haemorrhage } \\
\hline NRCMS & 1465 & $718(49.0)$ & 1.14 (0.97 to 1.35$)$ & 0.1063 & 1.27 (1.06 to 1.52$)$ & 0.0112 \\
\hline Self-payment & 263 & $112(42.6)$ & 0.88 (0.67 to 1.16$)$ & 0.3792 & 1.01 (0.75 to 1.34$)$ & 0.9709 \\
\hline UBMIS & 940 & $429(45.6)$ & Ref & & Ref & \\
\hline
\end{tabular}

*Adjusted for age, mRS score, family monthly income per capita, smoking, drinking, NIHSS score on administration, cerebrovascular disease type, previous stroke or TIA, heart failure/myocardial infarction, dyslipidaemia, hospital level, antiplatelets and anticoagulants in the Cox model with consideration of the hospital's cluster effect.

†Adjusted for age, mRS score, family monthly income per capita, drinking, NIHSS score on administration, previous stroke or TIA, heart failure/myocardial infarction, hypertension, diabetes mellitus, hospital level, antiplatelets and anticoagulants in the Cox model with consideration of the hospital's cluster effect.

$\ddagger$ Adjusted for age, sex, education, mRS score, cerebrovascular disease type, smoking, drinking, heart failure or myocardial infarction, diabetes mellitus, hospital level, antiplatelets and anticoagulants in the generalised equation estimation model. mRS, modified Rankin Scale; NIHSS, National Institutes of Health Stroke Scale; NRCMS, New Rural Cooperative Medical Scheme; Ref, reference; TIA, transient ischaemic attack; UBMIS, Urban Basic Medical Insurance Scheme. 
of urban or rural living. Therefore it is tough to determine if the differences can be attributed to insurance status as proposed, or to unmeasured factors including lifestyle issues or even ethnic/genetic variations. Although we could not have all these confounders controlled, we made a reasonable covariates list for adjustment, and the results were consistent with other studies.

\section{CONCLUSIONS}

In conclusion, in this nationwide registry study, we found that patients with stroke and TIA covered by NRMCS have a higher risk of 1-year all-cause death, stroke recurrence and disability than those covered by UBMIS, especially patients with ischaemic stroke. Further research aiming to assess the underlining reasons affecting resource accessibility and health service utilisation among different health insurance statuses would be helpful. Improving accessibility of health service for rural insurance patients with ischaemic stroke may improve stroke outcomes and lower the stroke burden in China, especially in rural regions.

\section{Author affiliations \\ ${ }^{1}$ China National Clinical Research Center for Neurological Diseases, Beijing, China ${ }^{2}$ Tiantan Clinical Trial and Research Center for Stroke, Department of Neurology, Beijing Tiantan Hospital, Capital Medical University, Beijing, China \\ ${ }^{3}$ Vascular Neurology, Department of Neurology, Beijing Tiantan Hospital, Capital Medical University, Beijing, China \\ ${ }^{4}$ Neuro-intensive Care Unit, Department of Neurology, Beijing Tiantan Hospital, Capital Medical University, Beijing, China \\ ${ }^{5}$ Center for Stroke, Beijing Institute for Brain Disorders, Beijing, China ${ }^{6}$ Beijing Key Laboratory of Translational Medicine for Cerebrovascular Disease, Beijing, China \\ ${ }^{7}$ Institute of Molecular Medicine, Yingjie Center, Peking University, Beijing, China ${ }^{8}$ Department of Neuropsychiatry and Behavioral Neurology and Clinical Psychology, Beijing Tiantan Hospital, Capital Medical University, Beijing, China}

Acknowledgements Beijing Shijitan Hospital; Peking University Third Hospital; Beijing Anzhen Hospital; Tianjin Medical University General Hospital; Tianjin Huanhu Hospital; Daqing Oil field General Hospital; Tangshan Shi Gongyin Hospital; Qinhuangdao Military Hospital; The Second Hospital of Hebei Medical University; Traditional and Western Medicine Integration Hospital of Cangzhou City, Hebei Province; Handan Shi Zhongxin Hospital; Handan No 1 Hospital; Kailuan General Hospital; Yuci District People's Hospital; Cardiovascular Disease Hospitals in Shanxi Province; JiShan People's Hospital; Inner Mongolia People's Hospital; Ulanqab Central Hospital; The First Affiliated Hospital of Dalian Medical University; Dalian Central Hospital; Dalian Economic and Technological Development District Hospital; Dalian Third People's Hospital; En'liang Hopital of Tai'an County; National Traditional Chinese Medicine (TCM) Thrombus Treatment Center of Liaoning Province; Jilin Oilfield General Hospital; The Second Clinical Medical College of Beihua University(The General Hospital of CNPC in Jilin); The First Clinical College of Jilin University; The Fourth Clinical College of Harbin Medical University; The Second Clinical College of Harbin Medical University; Shanghai Yangpu Area Center Hospital; Shanghai Yangpu Area Shidong Hospital; Shanghai First Municipal People's Branch Hospital; Shanghai Ruijin Hospital; The Sixth People's Hospital Affiliated to Shanghai Jiao Tong University; Huashan Hospital of Fudan University; Pudong New Area People's Hospital; Shanghai Renji Hospital Affiliated to Shanghai Jiao Tong University; The Affiliated Hospital of Nantong University Upper First-class Hospital; Drum Tower Hospital Affiliated to Nanjing University Medical School; The First Affiliated Hospital of Zhejiang University; The First Affiliated Hospital of Wenzhou Medical College; Zhoushan People's Hospital; The First Affiliated Hospital of Fujian Medical University; The First Hospital of Nanchang; Penglai People's Hospital; Liaocheng People's Hospital; Shandong Qilu Hospital; The First Affiliated Hospital of Henan College Of TCM; People's Hospital of Qi County; The First Clinical College of Zhengzhou University; Tongji Hospital, Tongji Medical College of HUST; Union Hospital, Tongji Medical College of HUST; Hubei Xinhua Hospital Upper First-class
Hospital; Chenzhou FirstPeople's Hospital; Xiangya Hospital of Centre-south University; The First Affiliated Hospital of Jinan University; Liwan Hospital of Guangzhou Medical College; Guangzhou First TMUNICIPAL People's Hospital; Guangdong People's Hospital; Dungun Municipal People's Hospital; The People's Hospital of Guangxi Zhuang Autonomous Region; Qiongshan district People's Hospital of Haikou City; The Second Affiliated Hospital of Chongqing Medical University; The First Affiliated Hospital of Chongqing Medical University; Southwest Hospital; Third Military Medical University; Shaanxi Provincial People's Hospital; The First Affiliated Hospital of Medical College of Xian Jiaotong University; The Second Affiliated Hospital of Lanzhou University; Qinghai Provincial People's Hospital; Traditional Chinese Medicine Hospital of Xinjiang Uygur Autonomous Region; People's Hospital of Xinjiang Uygur Autonomous Region; Hospital of Xinjiang Production \& Construction Corps; The Luhe Teaching hospital of the Capital Medical University; Beijing Haidian Hospital; First Hospital of Huairou District; Beijing Jingmei Group General Hospital; Beijing Pinggu Hospital; Central Hospital of Aviation Industry (361Hospital); Beijing Miyunxian Hospital; Liangxiang Hospital of Fangshan District, Beijing; The First Hospital of Fangshan District, Beijing; Beijing Chuiyangliu Hospital(Beijing Minimally Invasive Hospital); People's Hospital of Beijing Daxing District; China Rehabilitation Research Center; The 263 Hospital of the Chinese People's Liberation Army; The Central Hospital of China Aerospace Corporation; Dagang Hospital of Binhai New Area;CNOOC General Hospital; Tianjin Ninghe Hospital; The No.2 Hospital of Baoding;The Hospital of Yutian County; Second Affiliated Hospital of Xingtai Medical College; Traditional Chinese Medicine Hospital of Xinle; Shijiazhuang Central Hospital; Hengshui NO.5 People's Hospital; Hebei General Hospital; Hebei Union University Affiliated Hospital; Traditional Chinese Medicine Hospital of Qiu County; Affiliated Hospital of Hebei University; Cangzhou People's Hospital; Baoding N0.2 Central Hospital; Traditional Chinese Medicine Hospital of Yutian County; Tangshan No.9 Hospital; Shijiazhuang First Hospital; Qianan Yanshan Hospital; General Hospital of TISCO; The First Affiliated Hospital of Shanxi Medical University; Xinzhou People's Hospital; Shanxi Fenyang Hospital; Houma People's Hospital; General Hospital of Shanxi Fenxi Mining (Group) LLC; The First Affiliated Hospital of Baotou Medical College; Affiliated Hospital of Chifeng University; Ordos Central Hospital; Chifeng Municipal Hospital; Shengjing Hospital of China Medical University; Anshan Changda Hospital; Central People's Hospital of Xiuyan Manchu Autonomous County; Wafangdian Third Hospital; Panjin Second Hospital; The First Affiliated Hospital of Liaoning Medical University; Fengcheng Central Hospital; Jinzhou Central Hospital; Fuxin Central Hospital; General Hospital of Benxi Iron and Steel(GROUP); Anshan Central Hospital; Jilin Central Hospital; The 222 Hospital of the Chinese People's Liberation Army; People's Hospital of Tailai County; The Hospital of Changzheng Machine Tool Group; Heilongjiang Provincial Nongken General Hospital; The Third Affiliated Hospital of Qiqihar Medical University; Hongqi Hospital of Mudanjiang Medical University; Shanghai Changhai Hospital; Tongzhou District N0.3 People's Hospital of Nantong; The Affiliated Hospital of Xuzhou Medical College; The Second Affiliated Hospital of Xuzhou Medical College; The First People's Hospital of Xuzhou; Shuyang People's Hospital; The Second People's Hospital of Lianyungang; Subei People's Hospital; Second Traditional Chinese Hospital of Jiangsu Province; The Affiliated Hospital of Zhejiang University School of Medicine; Traditional and Western Medicine Integration Hospital of Wenzhou City; Taizhou First People's Hospital; Shaoxing Central Hospital; Beilun District People's Hospital; Linhai First People's Hospital; Lishui People's Hospital; Maanshan Shiqiye Hospital; WuhuNO.2 People's Hospital; Huainan Chaoyang Hospital; N0.2 Hospital Xiamen;Jiangxi Provincial People's Hospital; Fengcheng People's Hospital; N0.2 Hospital Nanchang; Linzi District People's Hospital; Weifang Traditional Chinese Hospital; The Second Affiliated Hospital of Shandong University of Traditional Chinese Medicine; Qianfoshan Hospital(Qianfoshan Hospital Affiliated to Shandong University); Shandong Police Hospital; People's Hospital of Huantal County; Qufu People's Hospital; Qingzhou People's Hospital; Traditional Chinese Medicine Hospital of Penglai; Linyi People's Hospital; Liaocheng Dongchangfu People's Hospital; People's Hospital of Donge County; Pingdingshan People's Hospital N0.1; Luoyang Central Hospital Affiliated to Zhengzhou University; People's Hospital of Zhecheng; Shangqiu N0.3 People's Hospital; Nanyang Second General Hospital; Luohe Central Hospital; Henan Province Hospital of TCM; Xinxiang Central Hospital; Xiangyang Central Hospital; General Hospital of The Yangtze River Shipping, Wuhan Brain Hospital; Qianjiang Central Hospital; Hubei Provincial Zhongshan Hospital; Wuhan General Hospital of Guangzhou Military; Hubei Space Hospital; Hunan Provincial People's Hospital; Nanfang Hospital; Southern Medical University; Yangjiang Hospital; Shenzhen People's Hospital; The Second Affiliated Hospital of Guangzhou Medical University; The Affiliated Hospital of Guangdong Medical College; Zhujiang Hospital of Southern Medical University; The People's Hospital of Wuzhou; The First Affiliated Hospital of Guangxi Medical University; The Hospital of Gongxi Liuzhou Iron and Steel(GROUP) Company; Hainan General Hospital; Hainan Provincial Nongken General Hospital; Hainan Province Nongken 
Sanya Hospital; Geriatrics Hospital of Hainan Province; Traditional Chinese Medicine Hospital of Haikou; Xinqiao Hospital of Third Military University of Chinese P.L.A People's Hospital of Deyang City; West China Hospital,Sichuan University; Xindu District People's Hospital of Chengdu; Wenjiang District People's Hospital of Chengdu; Zunyi First People's Hospital; Bijie First Municipal People's Hospital; People's Hospital of Yuxi City; Tibet Autonomous Region People's Hospital; Tibet Autonomous Region Second People's Hospital; Central Hospital of China Railway 20 Bureau Group Corporation; Yanan University Affiliated Hospital; Chang'an Hospital; Xi'an 141 Hospital; Xi'an N0.1 Hospital; The Second Affiliated Hospital of Lanzhou University; Qinghai University Affiliated Hospital; Zhongwei People's Hospital; General Hospital of Ningxia Medical University; The Third People's Hospital of Ningxia; The First Affiliated Hospital of Shihezi University School of Medicine; Xinjiang Fukang People's Hospital.

Contributors $\mathrm{H}-\mathrm{QG}$ had full access to all of the data in the study and takes responsibility for the integrity of the data and the accuracy of the data analysis. $\mathrm{Y}$-LW and Y-JW initiated the study and obtained the funding. $\mathrm{H}-\mathrm{QG}$ and Z-XL did the concept and design of this study. $\mathrm{H}-\mathrm{QG}$ also performed the statistical analysis and drafted the manuscript. All the other authors including X-QZ, L-PL, HL, C-JW, XY, Z-ZR, C-XW and Y-SP participated in the acquisition, analysis or interpretation of data, and critical revision of the manuscript for valuable intellectual content.

Funding This work was supported by grants from the Ministry of Science and Technology of the People's Republic of China (2006BA101A11, 2008ZX09312-008, 2011BAI08B01, 2011BAI08B02, 2012ZX09303, 2013BAI09B14, 2013BAI09B03, 2015BAl12B02 and 2015BAl12B04), Beijing Municipal Commission of Science and Technology (D15110700200000 and Z161100000516223), Beijing Institute for Brain Disorders (BIBD-PXM2013_014226_07_000084) and Beijing Tiantan Hospital Seeding Initiative.

Competing interests None declared.

Patient consent Obtained.

Ethics approval The study was approved by the Central Institutional Review Board at Beijing Tiantan Hospital.

Provenance and peer review Not commissioned; externally peer reviewed.

Data sharing statement No additional data are available.

Open access This is an open access article distributed in accordance with the Creative Commons Attribution Non Commercial (CC BY-NC 4.0) license, which permits others to distribute, remix, adapt, build upon this work non-commercially, and license their derivative works on different terms, provided the original work is properly cited and the use is non-commercial. See: http://creativecommons.org/ licenses/by-nc/4.0/

(c) Article author(s) (or their employer(s) unless otherwise stated in the text of the article) 2018. All rights reserved. No commercial use is permitted unless otherwise expressly granted.

\section{REFERENCES}

1. Levy H, Meltzer D. The impact of health insurance on health. Annu Rev Public Health 2008;29:399-409.

2. Dor A, Sudano J, Baker DW. The effect of private insurance on the health of older, working age adults: evidence from the health and retirement study. Health Serv Res 2006;41(3 Pt 1):759-87.

3. Hadley J, Waidmann T. Health insurance and health at age 65: implications for medical care spending on new Medicare beneficiaries. Health Serv Res 2006;41:429-51.

4. Sun Y, Gregersen H, Yuan W. Chinese health care system and clinica epidemiology. Clin Epidemiol 2017;9:167-78.

5. Blumenthal $D$, Hsiao W. Lessons from the East--China's rapidly evolving health care system. N Engl J Med 2015;372:1281-5.

6. Freeman JD, Kadiyala S, Bell JF, et al. The causal effect of health insurance on utilization and outcomes in adults: a systematic review of US studies. Med Care 2008;46:1023-32.

7. McManus M, Ovbiagele B, Markovic D, et al. Association of Insurance Status with Stroke-Related Mortality and Long-term Survival after Stroke. J Stroke Cerebrovasc Dis 2015;24:1924-30.

8. Kreindel S, Rosetti R, Goldberg R, et al. Health insurance coverage and outcome following acute myocardial infarction. A communitywide perspective. Arch Intern Med 1997;157:758-62.

9. Shen JJ, Washington EL. Disparities in outcomes among patients with stroke associated with insurance status. Stroke 2007;38:1010-6.
10. Zhang $X$, Zhang $Y$, Xiao $X$, et al. The relation between health insurance and management of hypertension in Shanghai, China: a cross-sectional study. BMC Public Health 2016;16:959.

11. Liu B, Yan H, Guo R, et al. The basic social medical insurance is associated with clinical outcomes in the patients with ST-elevation myocardial infarction: a retrospective study from Shanghai, China. Int J Med Sci 2014;11:905-17.

12. Yan R, Li W, Yin L, et al. Cardiovascular diseases and risk-factor burden in urban and rural communities in high-, middle-, and lowincome regions of china: a large community-based epidemiologica study. J Am Heart Assoc 2017;6:e004445.

13. Xu F, Ah Tse L, Yin X, et al. Impact of socio-economic factors on stroke prevalence among urban and rural residents in Mainland China. BMC Public Health 2008;8:170-70.

14. Zhang $\mathrm{XH}$, Guan T, Mao J, et al. Disparity and its time trends in stroke mortality between urban and rural populations in China 1987 to 2001: changing patterns and their implications for public health policy. Stroke 2007;38:3139-44

15. Johnston SC, Mendis S, Mathers CD. Global variation in stroke burden and mortality: estimates from monitoring, surveillance, and modelling. Lancet Neurol 2009;8:345-54.

16. Strong K, Mathers C, Bonita R. Preventing stroke: saving lives around the world. Lancet Neurol 2007;6:182-7.

17. Wang W, Jiang $B$, Sun $H$, et al. Prevalence, incidence, and mortality of stroke in china: results from a nationwide population-based survey of 480687 adults. Circulation 2017;135:759-71.

18. The Ministry of Health of the People's Republic of China. 2010 Annual chinese healthcare statistics. Beijing: China Union Medical University Publication, 2010.

19. Yang X, Li Z, Zhao X, et al. Use of warfarin at discharge among acute ischemic stroke patients with nonvalvular atrial fibrillation in China. Stroke 2016;47:464-70.

20. Li Z, Wang C, Zhao X, et al. Substantial progress yet significant opportunity for improvement in stroke care in China. Stroke 2016;47:2843-9.

21. Wang Y, Cui L, Ji X, et al. The China National Stroke Registry for patients with acute cerebrovascular events: design, rationale, and baseline patient characteristics. Int J Stroke 2011;6:355-61.

22. Rankin J. Cerebral vascular accidents in patients over the age of 60 . II. Prognosis. Scott Med J 1957;2:200-15.

23. Lyden P, Brott T, Tilley B, et al. Improved reliability of the NIH Stroke Scale using video training. NINDS TPA Stroke Study Group. Stroke 1994;25:2220-6.

24. Wienke A. Frailty models in survival analysis: CRC Press, 2010.

25. Fine JP, Gray RJ. A proportional hazards model for the subdistribution of a competing risk. J Am Stat Assoc 1999;94:496-509.

26. Hardin JW. Generalized estimating equations (GEE): Wiley Online Library, 2005

27. Meng Q, Fang $\mathrm{H}$, Liu X, et al. Consolidating the social health insurance schemes in China: towards an equitable and efficient health system. Lancet 2015;386:1484-92.

28. Gezmu T, Gizzi MS, Kirmani JF, et al. Disparities in acute stroke severity, outcomes, and care relative to health insurance status. $J$ Stroke Cerebrovasc Dis 2014;23:e93-e98.

29. Fargen KM, Neal D, Blackburn SL, et al. Health disparities and stroke: the influence of insurance status on the prevalence of patient safety indicators and hospital-acquired conditions. J Neurosurg 2015;122:870-5

30. Hasan O, Orav EJ, Hicks LS. Insurance status and hospital care for myocardial infarction, stroke, and pneumonia. $J$ Hosp Med 2010;5:452-9.

31. James ML, Grau-Sepulveda MV, Olson DM, et al. Insurance status and outcome after intracerebral hemorrhage: findings from Get With The Guidelines-stroke. J Stroke Cerebrovasc Dis 2014;23:283-92.

32. Fonarow GC, Saver JL, Smith EE, et al. Relationship of national institutes of health stroke scale to 30-day mortality in medicare beneficiaries with acute ischemic stroke. J Am Heart Assoc 2012;1:42-50.

33. Addo J, Ayerbe L, Mohan KM, et al. Socioeconomic status and stroke: an updated review. Stroke 2012;43:1186-91.

34. Currie J, Decker S, Lin W. Has public health insurance for older children reduced disparities in access to care and health outcomes? $J$ Health Econ 2008;27:1567-81.

35. Franks P, Clancy CM, Gold MR. Health insurance and mortality. Evidence from a national cohort. JAMA 1993;270:737-41.

36. Skolarus LE, Meurer WJ, Burke JF, et al. Effect of insurance status on postacute care among working age stroke survivors. Neurology 2012;78:1590-5.

37. Mclntyre D, Ranson MK, Aulakh BK, et al. Promoting universal financial protection: evidence from seven low- and middle-income 
countries on factors facilitating or hindering progress. Health Res Policy Syst 2013;11:36.

38. McIntyre D, Garshong B, Mtei G, et al. Beyond fragmentation and towards universal coverage: insights from Ghana, South Africa and the United Republic of Tanzania. Bull World Health Organ 2008;86:871-6.

39. The State Council of the People's Republic of China. http://www.gov. cn/xinwen/2017-12/14/content_5246738.htm 